\title{
Plantas Alimenticias que se ofertan en los mercados de la ciudad de León
}

\author{
Angélica Maria Guevara Guevara ${ }^{1}$, Ricardo M. Rueda Pereira ${ }^{1^{*}}$ \\ 1. Departamento de Biología, Facultad de Ciencias y Tecnología, Universidad Nacional Autónoma de Nicaragua, León.
}

\section{RESUMEN}

El propósito de este estudio es conocer las plantas alimenticias que se ofertan en los cuatro mercados de la ciudad de León, Terminal, Estación, Central y mercadito Sutiava, en el periodo comprendido de mayo 2006 a mayo 2007 investiga sobre procedencia, situación de mercadeo y usos de estas plantas. Se realizaron visitas a los diferentes mercados dos veces por mes (inicio y final) para conocer que plantas ingresan y cuales son permanentes en los diferentes meses. La recopilación de datos se hizo mediante el uso de entrevistas etnobotánicas aplicadas a los vendedores de plantas de los mercados existentes en la ciudad. Se encontraron un total de 103 especies, 28 especies de estas plantas son nativas y 75 introducidas, las cuales están agrupadas en 43 familias de estas 6 son monocotiledóneas y 37 dicotiledóneas y un total de 79 Géneros. Las familias mas abundantes son: Rutaceae, Cucurbitaceae, Apiaceae, Poaceae, Myrtaceae y Solanaceae. Con respecto a disponibilidad 60 plantas se encuentran en los mercados durante todo el año, en el mes de agosto ingresan más plantas en el año donde se reportan 27 especies, y el mes en que menos ingresan es febrero con 9 especies.

Palabras claves: Planta, alimenticia, mercado, etnobotánica.

\section{INTRODUCCIÓN}

Las plantas que son cultivadas o explotadas por el hombre constituyen un número muy pequeño de especies en comparación con las que existen en las comunidades naturales y su utilización disminuye a un más cada día, pues se va perdiendo el conocimiento tradicional acerca del uso de muchas plantas. ${ }^{[1]}$

En el medio tropical precisamente en América Central muchas plantas nativas crecen prácticamente en estado silvestre, sin embargo, estas son objeto de recolección por parte de algunos campesinos que sobreviven del comercio de estas plantas, entre estas se pueden citar: zapotes, nísperos, anonas, jocotes, mamones, nancites, cálalas, aguacates, etc. Hoy en día estas plantas representan un componente importante en la dieta de las poblaciones latinoamericanas y además una alternativa de fuentes de ingresos.

Durante mucho tiempo la producción silvestre y casera de las plantas alimenticias han sido suficiente para abastecer a los mercados nacionales, donde el crecimiento interno de la población y la necesidad de encontrar nuevas fuentes de ingresos, con la exportación de productos tropicales han originado la creación de plantaciones de carácter comercial. ${ }^{[2]}$

En Nicaragua existe una carencia de información básica sobre plantas alimenticias, hay poca experiencia, los trabajos de investigación son limitados, poco divulgados y hay poco acceso de información fuera del área centroamericana. Ante esta situación los campesinos y futuros productores que sobreviven del comercio de estas plantas esenciales en el consumo humano se enfrentan a muchos problemas por desconocer las necesidades de los mercados, las variedades de mayor demanda y sobre todo al no dominar las practicas culturales más elementales. ${ }^{[2]}$

En el país, la producción de frutas apetitosas y vegetales frescos ha aumentado en los últimos años en la zona norte del país, la cual ha sido una fuente de ingresos para los campesinos y pequeños productores que sobreviven del comercio de estas plantas alimenticias. Nicaragua tiene una gran diversidad de frutas, vegetales, hortalizas y es necesario generar una cultura de mayor consumo de estas plantas para mantener y mejorar nuestra salud. Además, estas frutas nicaragüenses conocidas y algunas exóticas han logrado ser una fuente de inagotables experiencias de gustos tanto para visitantes extranjeros y para los mismos nacionales. ${ }^{[2]}$

\section{DISEÑO METODOLÓGICO}

El estudio se realizó en el período comprendido de mayo 2006 a mayo 2007, en los cuatro mercados existentes en la ciudad de León: Mercado La Terminal, Mercado La Estación, Mercado Central y Mercadito de Sutiava. 
Para la recopilación de datos se realizaron visitas a los mercados al inicio y al final de cada mes para conocer las plantas alimenticias, que se ofertan e ingresan en los mercados en los diferentes meses y cuales de estas especies eran permanentes durante todo el año, asi como también obtener información sobre los precios de las mismas a lo largo del año.

La información se obtuvo mediante el uso de entrevistas las cuales fueron aplicadas a los diferentes vendedores de plantas de los cuatro mercados de la ciudad, las encuestas fueron aplicadas por cada especie encontrada en los mercados durante el período de investigación, estas contenían preguntas abiertas y cerradas donde se reflejaba el conocimiento que tienen los vendedores sobre el producto que ofertan.

Las entrevistas incluían preguntas tales como: la procedencia del producto que se oferta, valor económico, auges de ventas, forma de venta, tiempo de permanencia en los mercados del producto ofertado, época de mayores ventas (estación seca o lluviosa) y los diferentes usos de las plantas alimenticias.

\section{RESULTADOS Y DISCUSIÓN}

De acuerdo a los datos obtenidos durante la recopilación de información en los diferentes mercados de la ciudad de León en el periodo de estudio, se reportaron un total de 103 especies agrupadas en 6 familias, 14 géneros y 16 especies de Monocotiledóneas y 37 familias, 65 Géneros y 87 especies de Dicotiledóneas.

Es importante señalar que de los cuatro mercados que existen en la ciudad de León en el mercado La Terminal se encontró una mayor variedad de productos alimenticios incluyendo especies que a veces es difícil encontrarlas en los demás mercados, por lo cual a este se le ha considerado como el mayoreo y principal distribuidor de plantas alimenticias para los demás mercados de la ciudad.

El análisis de las encuestas realizadas revela que la mayoría de las plantas ofertadas proceden del departamento de León incluyendo sus municipios y comarcas aledañas como las principales abastecedoras de plantas alimenticias. Los productores de estas plantas tienen la opción de vender directamente sus productos en los mercados de la ciudad con la posibilidad de que estos pueda ser ofertado al consumidor o a revendedores locales. En este estudio, también, se reporta que la mayoría de las plantas ofertadas son frutas y que 60 especies de estas plantas alimenticias se pueden encontrar en los mercados durante todo el año. A estas plantas alimenticias, también, se les atribuyen propiedades medicinales los cuales fueron reportados por los vendedores de los cuatro mercados.

\section{CONCLUSIONES}

Las plantas estudiadas en los mercados de la ciudad de León se encuentran distribuidas en 43 familias, 79 géneros para un total de 103 especies, siendo las familias Rutaceae, Cucurbitaceae, Apiaceae y Poaceae las que presentaron el mayor número de especies. En los Mercados Central, Estación, Terminal y Sutiava, la mayor cantidad de plantas proceden del departamento de León.

Del total de plantas alimenticias reportadas en el estudio 60 se encuentran en los mercados durante los 12 meses del año, Nancite, Berenjena y Melón se encontraron disponibles en los mercados durante 10 meses, Chirimoya, Malanga y Soncoya ingresaron solamente 1 mes en el año de estudio. Las frutas son las que gozan de una mayor cantidad de especies agrupadas por categorías alimenticias.

Con respecto a la época de mayores ventas estas se mantienen con las diferentes especies durante todo el año auque hay algunas plantas que se ofertan solamente por temporadas. La principal forma de venta de las plantas es por unidad. De acuerdo, a los resultados obtenidos se puede observar la gran cantidad de plantas alimenticias que se venden en los mercados, así como, también, que estas plantas tienen otros usos como medicinales los cuales fueron reportados por los vendedores.

\section{REFERENCIAS BIBLIOGRÁFICAS}

1. FAO/Organización de las Naciones Unidas para la Alimentación yAgricultura/Lasplantasqueutilizamos/ documentos/enlínea/consultado 26/06/2006/ disponible en: http://WWW.fao.org.

2. Barbeau G, 1990. Frutas Tropicales de Nicaragua. 1ra Edición. Editorial Ciencias Sociales. Managua, Nicaragua.

\section{ANEXOS}

ANEXO 1. Nombres científicos y comunes de las Plantas Alimenticias que se ofertan en los mercados de la ciudad de León. 
Nombres científicos y comunes de las Plantas Alimenticias que se ofertan en los mercados de la ciudad de León.

\begin{tabular}{|c|c|}
\hline Nombre científico & Nombre común \\
\hline Annona cherimola Mill. & Chirimoya \\
\hline Annona reticulata $L$. & Anona \\
\hline Annona muricata $L$. & Guanábana \\
\hline $\begin{array}{l}\text { Annona purpurea Moç. \& Sessé } \\
\text { ex Dunal. }\end{array}$ & Soncoya \\
\hline Anacardium occidentale $L$. & Marañon \\
\hline Mangifera indica $L$. & Mango \\
\hline Spondias purpúrea L. & Jocote \\
\hline Apium graveolens $L$. & Apio \\
\hline Cuminum cyminum L. & Comino \\
\hline Daucus carota $L$. & Zanahoria \\
\hline Eryngium foetidum $L$. & Culantro \\
\hline $\begin{array}{l}\text { Petroselinum crispum (Mill.) } \\
\text { Nyman }\end{array}$ & Perejil \\
\hline Pimpinela anisum $L$ & Anís \\
\hline Colocasia esculenta (L) Schott & Malanga \\
\hline Xanthosoma violaceum Schott & Quequisque \\
\hline Acrocomia mexicana Karw. & Coyol \\
\hline Bactris major Jacq. & Coyolito \\
\hline Cocos nucifera $L$. & Coco \\
\hline Lactuca sativa $L$. & Lechuga \\
\hline Crescentia alata Kunth & Semilla de jicaro \\
\hline Bixa orellana $L$. & Achiote \\
\hline Brassica oleraceae L. & Repollo \\
\hline $\begin{array}{c}\text { Brassica oleraceae var. capitata } \\
\text { L. }\end{array}$ & Coliflor \\
\hline $\begin{array}{c}\text { Brassica oleraceae var. italica } \\
L .\end{array}$ & Brócoli \\
\hline Brassica nigra $L$. & Mostaza \\
\hline Rhafanus sativus $L$. & Rábano \\
\hline Ananas comosus (L.) Merr. & Piña \\
\hline Bromelia pinguin $L$. & Piñuela \\
\hline $\begin{array}{c}\text { Hylocereus undatus (Haw.) } \\
\text { Britton \& Rose }\end{array}$ & Pitahaya \\
\hline
\end{tabular}

\begin{tabular}{|c|c|}
\hline $\begin{array}{c}\text { Citrullus lanatus (Thumb.) } \\
\text { Matsum. \& Nakai }\end{array}$ & Sandia \\
\hline Cucumis melo L. & Melón \\
\hline Cucumis sativus $L$. & Pepino \\
\hline Cucurbita moschata Duchesne & Ayote \\
\hline Cucurbita pepo L. & Pipían \\
\hline Sechium edule (Jacq.) Sw. & Chayote \\
\hline Sicana odorifera (Vell.) Naudin & Cohombro \\
\hline Tamarindus indica $L$. & Tamarindo \\
\hline Carica papaya $L$. & Papaya \\
\hline Beta vulgaris $L$. & Remolacha \\
\hline $\begin{array}{c}\text { Couepia polyandra (Kunth) } \\
\text { Rose }\end{array}$ & Zapote amarillo \\
\hline Chrysobalanus icaco $L$. & Icaco \\
\hline $\begin{array}{c}\text { Licania platypus (Hemsl.) } \\
\text { Fritsch. }\end{array}$ & Sonzapote \\
\hline Mammea americana $L$. & Mamey \\
\hline Manihot esculenta Crantz. & Yuca \\
\hline Phyllanthus acidus (L.) Skeels & Grosella \\
\hline Arachis hypogaea L. & Mani \\
\hline Glycine max (L.) Merr. & Soya \\
\hline Phaseolus vulgaris $L$. & Frijol \\
\hline Vigna unguiculata (L.) Walp. & Frijol de vara \\
\hline $\begin{array}{c}\text { Cinnamomun zeylanicum } \\
\text { Breyne }\end{array}$ & Canela \\
\hline Persea americana Mill. & Aguacate \\
\hline Salvia hispánica L. & Chia \\
\hline Mentha x piperita $L$. & Hierbabuena \\
\hline $\begin{array}{l}\text { Plectranthus amboinicus } \\
\text { (Lour.) Spreng. }\end{array}$ & Oregano \\
\hline Allium cepa $L$. & Cebolla \\
\hline \multicolumn{2}{|l|}{ Allium sativum $L$. } \\
\hline Linum usitatissimum L. & Linaza \\
\hline $\begin{array}{l}\text { Byrsonimia crassifolia (L). } \\
\text { Kunth }\end{array}$ & Nancite \\
\hline Hibiscus sabdariffa $L$. & Rosa de jamaica \\
\hline Inga paterno Harms. & Guava \\
\hline Myristica fragans Houtt. & Nuez moscada \\
\hline $\begin{array}{c}\text { Artocarpus altilis (Park.) } \\
\text { Fosberg }\end{array}$ & Semilla de pan \\
\hline
\end{tabular}


Nombres científicos y comunes de las Plantas Alimenticias que se ofertan en los mercados de la ciudad de León.

\begin{tabular}{|c|c|}
\hline Musa acuminata Colla & Banano \\
\hline Musa x paradisiaca L. & Platano \\
\hline $\begin{array}{c}\text { Eugenia caryophyllata Merr. \& } \\
\text { Perry }\end{array}$ & Clavo de olor \\
\hline Pimienta dioica (L.) Merr. & Pimienta de chapa \\
\hline Psidium guajava L. & Guayaba \\
\hline $\begin{array}{c}\text { Psidium friedrichsthalianum } \\
\text { (O. Berg) Nied. }\end{array}$ & Guayaba de fresco \\
\hline $\begin{array}{c}\text { Syzygium malaccense (L.) } \\
\text { Merr. \& Perry }\end{array}$ & Pera de agua \\
\hline Passiflora edulis Sims & Calala \\
\hline Passiflora quadrangularis $L$. & Granadilla \\
\hline Sesamum indicum $L$. & Ajonjoli \\
\hline Avena sativa $L$. & Avena \\
\hline Hordeum vulgare L. & Cebada \\
\hline Oryza sativa L. & Arroz \\
\hline Saccharum officinarum $L$. & Caña de azucar \\
\hline Sorghum vulgare Pers. & Sorgo \\
\hline Zea mays L. & Maiz \\
\hline Coccoloba caracasana Meissn. & Papaturro \\
\hline Malus comunis $L$. & Manzana \\
\hline
\end{tabular}

\begin{tabular}{|c|c|}
\hline Coffea arabica $L$. & Café \\
\hline Morinda citrifolia $L$. & Noni \\
\hline Citrus $X$ aurantium $L$. & Naranja agria \\
\hline $\begin{array}{c}\text { Citrus aurantifolia (Christm.) } \\
\text { Swingle }\end{array}$ & Limon agrio \\
\hline Citrus limetta Risso & Limon dulce \\
\hline Citrus maxima (Burm.) Merr. & Toronja \\
\hline Citrus paradisi Macf. & Grapefruit \\
\hline Citrus reticulata Blanco & Mandarina \\
\hline Citrus sinensis (L.) Osbek. & Naranja dulce \\
\hline Melicoccus bijugatus Jacq. & Mamon \\
\hline Nephelium lappaceum L. & Mamon chino \\
\hline Chrysophyllum cainito $L$. & Caimito \\
\hline Manilkara zapota (L.) P. Royen & Nispero \\
\hline $\begin{array}{c}\text { Pouteria sapota (Jacq.) H.E. } \\
\text { Moore \& Stearn. }\end{array}$ & Sapote \\
\hline Theobroma cacao $L$. & Cacao \\
\hline Capsicum annuum L. & Chile, chiltomo \\
\hline Lycopersicun esculentum Mill. & Tomate \\
\hline Solanum melongena $L$. & Berenjena \\
\hline Solanum tuberosum $L$. & Papa \\
\hline Averrhoa bilimbi L. & Mimbro \\
\hline Averrhoa carambola L. & Melocoton \\
\hline Vitis vinifera $L$. & Uva \\
\hline Zingiber officinale Roscoe & Gengibre \\
\hline
\end{tabular}

Fotos de plantas alimenticias que se ofertan en los mercados de la ciudad de León.
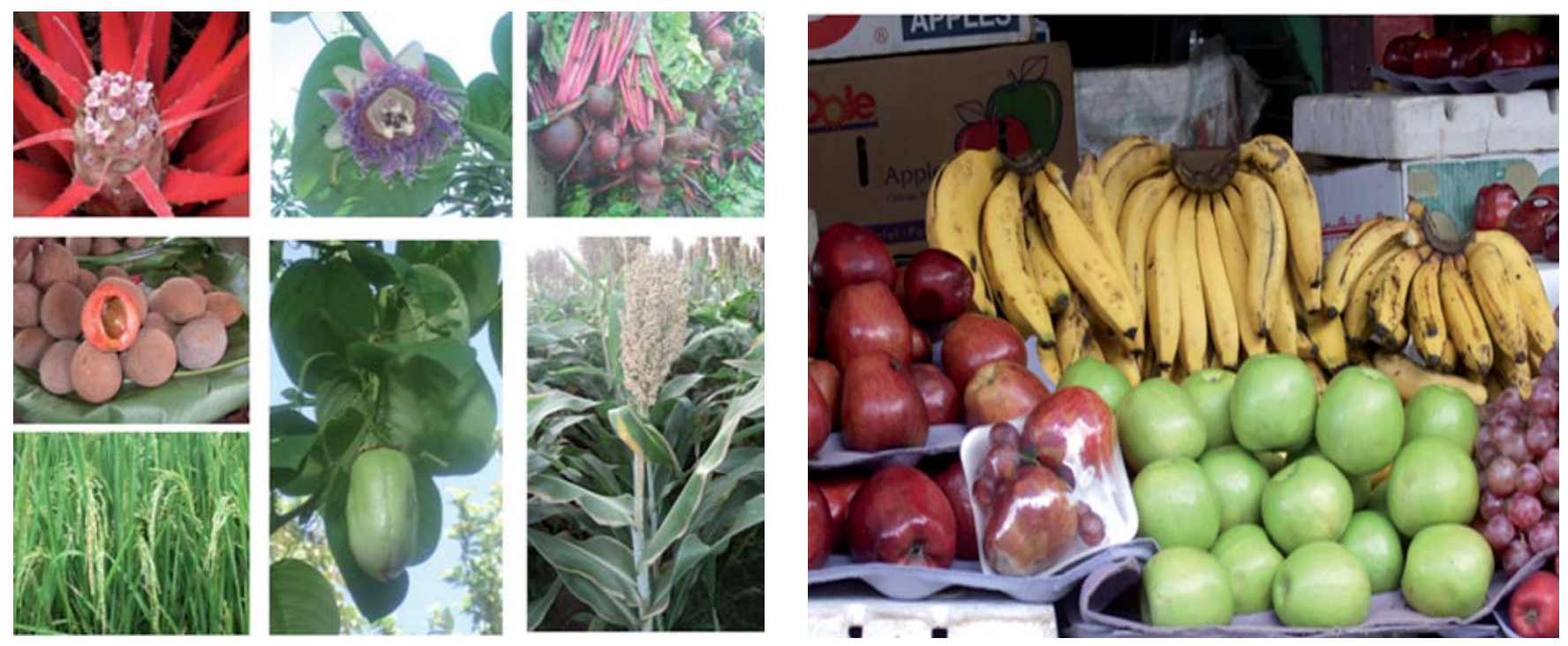

Fotos: Angélica María Guevara Guevara 